\title{
Repurposing benzbromarone for pulmonary arterial hypertension: can channelling the past deliver the therapy of the future?
}

\author{
Anne L. Theilmann and Mark L. Ormiston \\ Affiliation: Queen's University, Depts of Biomedical and Molecular Sciences, Medicine and Surgery, Kingston, \\ ON, Canada. \\ Correspondence: Mark L. Ormiston, Queen's University, Depts of Biomedical and Molecular Sciences, \\ Medicine and Surgery, Room A209, Botterell Hall, 18 Stuart Street, Kingston, Ontario K7L 3N6, Canada. \\ E-mail: mark.ormistondqqueensu.ca
}

@ERSpublications

A new study identifies increases in the calcium activated chloride channel, TMEM16A, in the pulmonary arteries of IPAH patients and proposes the repurposing of benzbromarone, a non-specific inhibitor of the channel, as a novel therapy for the disease http://bit.ly/2ITN4ul

Cite this article as: Theilmann AL, Ormiston ML. Repurposing benzbromarone for pulmonary arterial hypertension: can channelling the past deliver the therapy of the future?. Eur Respir J 2019; 53: 1900583 [https://doi.org/10.1183/13993003.00583-2019].

One of the most well-established hallmarks of pulmonary arterial hypertension (PAH) is the unique cellular phenotype of the pulmonary arterial smooth muscle cells (PASMCs) from PAH patients. This phenotype, which is defined by hypercontractility, excessive proliferation, apoptosis resistance and aerobic glycolysis, is intimately linked with plasma membrane depolarisation and elevated concentrations of cytosolic calcium $\left(\left[\mathrm{Ca}^{2+}\right]_{\text {cyt }}\right)[1-3]$.

Over the past two decades, multiple studies have identified the altered expression or regulation of a variety of cation channels that contribute to this depolarised PASMC phenotype in PAH. These changes include the increased expression of store-operated $\mathrm{Ca}^{2+}$ channels, including the canonical transient receptor potential (TrpC) channel, TrpC6 [4], and a reduction in voltage-gated potassium channels $\left(\mathrm{K}_{\mathrm{v}}\right)$, such as $\mathrm{K}_{\mathrm{v}} 1.5$ [5], which enable the efflux of $\mathrm{K}^{+}$ions from the intracellular space. Together, increased $\mathrm{Ca}^{2+}$ influx and reduced $\mathrm{K}^{+}$efflux contribute to membrane depolarisation and a further elevation of $\left[\mathrm{Ca}^{2+}\right]_{\mathrm{cyt}}$ via the opening of voltage-dependent calcium channels (figure 1). More recently, genetic screening of both heritable PAH families and idiopathic PAH (IPAH) patient cohorts enabled the identification of heterozygous loss-of-function mutations in $K C N K 3$, the gene encoding the TASK-1 potassium channel, in both patient populations $[6,7]$. KCNK3 is decreased in the monocrotaline rat model of PAH and its inhibition was found to induce aberrant pulmonary vascular cell proliferation in control rats [8]. Moreover, KCNK3 silencing promotes PASMC membrane depolarisation in vitro, offering yet another mediator of the diseased cellular phenotype in PAH [9].

In comparison to this thoroughly defined role for cation handling in $\mathrm{PAH}$, the potential contribution of anion channels to disease has received relatively little attention. To date, this work has largely been limited to studies of the $\mathrm{Ca}^{2+}$ activated $\mathrm{Cl}^{-}$channel, TMEM16A, which is upregulated in the PASMCs of rats exposed to either chronic hypoxia or monocrotaline and serves as a critical contributor to PASMC 
Healthy

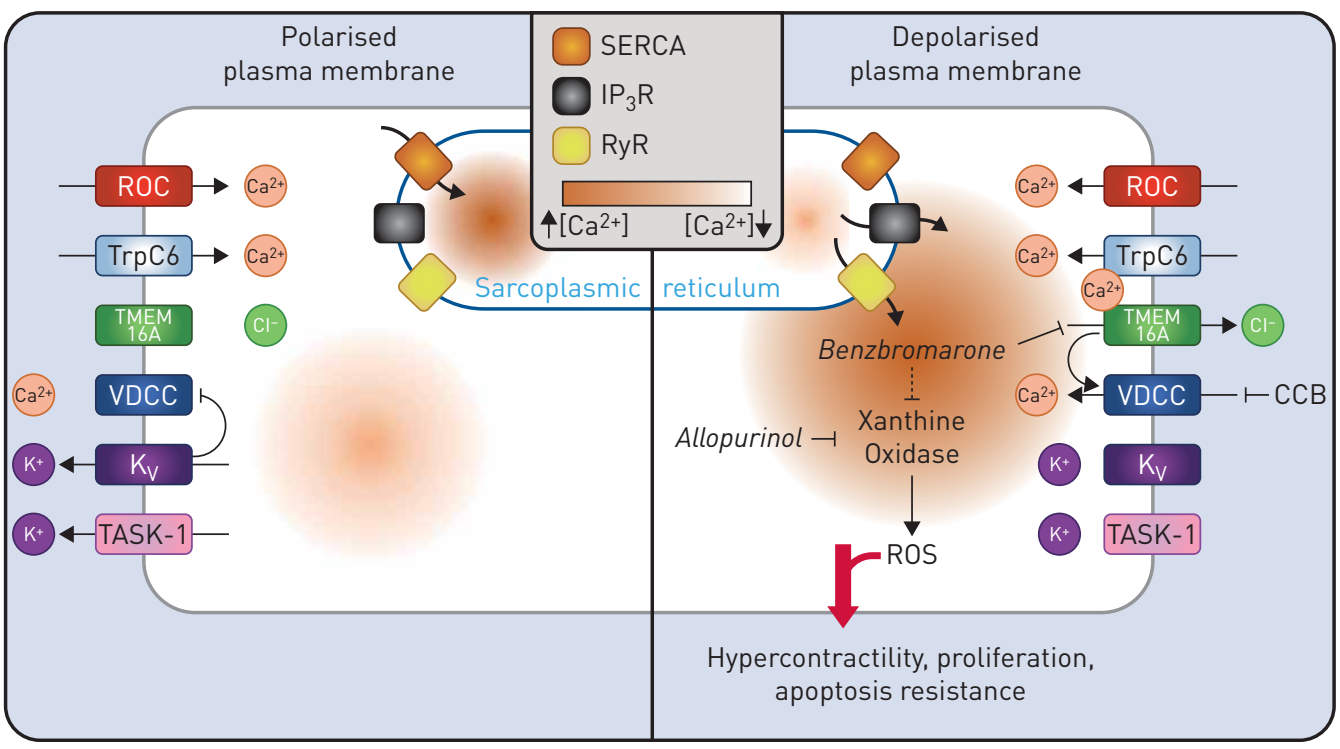

FIGURE 1 Schematic diagram summarising altered ion channel function in pulmonary arterial smooth muscle cells (PASMCs) from pulmonary arterial hypertension (PAH) patients and the proposed role for benzbromarone in reversing the cellular phenotype of disease. In PAH, store operated calcium channels, such as the canonical transient receptor potential $(\operatorname{TrpC})$ channel $\operatorname{TrpC} 6$, are upregulated while potassium channels, including TASK-1 and voltage-gated potassium channels $\left(\mathrm{K}_{\mathrm{v}}\right)$, are downregulated, leading to increased levels of cytosolic calcium and PASMC membrane depolarisation. These changes drive the activation of voltage-dependent calcium channels (VDCCs). Elevated expression of the $\mathrm{Ca}^{2+}$ activated $\mathrm{Cl}^{-}$ channel, transmembrane member 16A (TMEM16A), results in further PASMC membrane depolarisation, hypercontractility, proliferation and apoptosis resistance. Treatment of PASMCs with benzbromarone is believed to block TMEM16A, restoring membrane polarisation and reversing the PAH PASMC phenotype. However, benzbromarone, like allopurinol, may also enact therapeutic benefits through its actions on xanthine oxidase. CCB: calcium channel blockers; $\mathrm{IP}_{3} \mathrm{R}$ : inositol trisphosphate-sensitive receptor; ROC: receptor operated channel; RyR: ryanodine receptor; ROS: reactive oxygen species; SERCA: sarcoplasmic/endoplasmic reticulum calcium ATPase.

depolarisation and pulmonary artery hypercontractility in both models of disease $[10,11]$. Interestingly, studies in airway epithelial cells have shown that $\mathrm{Ca}^{2+}$ activated $\mathrm{Cl}^{-}$channels, which enable $\mathrm{Cl}^{-}$efflux in response to sub-micromolar increases in $\left[\mathrm{Ca}^{2+}\right]_{\mathrm{cyt}}$, can function in tandem with TrpC6, providing a possible link between increased TMEM16A activity and a known actor in the depolarisation of PAH PASMCs [12]. Despite this compelling evidence, work supporting an important role for TMEM16A, or other anion channels, in the pathogenesis of PAH has yet to move beyond animal models.

In the current issue of the European Respiratory Journal, PAPP et al. [13] address this limitation using a targeted screen of anion channel and transporter gene expression in laser capture microdissected pulmonary arteries from the lungs of IPAH patients and healthy control subjects. The authors identify TMEM16A as the only anion channel that is significantly upregulated in IPAH pulmonary arteries and demonstrate a central role for this channel in PASMC depolarisation. While these studies provide essential insight into the cellular pathobiology of the human condition, much of the value of the work by PAPP et al. [13] comes from their introduction of benzbromarone, a non-specific inhibitor of TMEM16A, as a potential PAH therapy.

Benzbromarone is a uricosuric agent and an inhibitor of xanthine oxidase that was first introduced in the 1970s as a treatment for gout [14]. It was not until 2012 that the drug was also identified, through a high-throughput screen, as an inhibitor of TMEM16A at micromolar concentrations [15]. PAPP et al. [13] demonstrate that benzbromarone restores membrane polarisation and blocks the platelet-derived growth factor-induced proliferation of PAH PASMCs in vitro, while preventing the development of pulmonary hypertension in vivo in both the chronic hypoxia mouse and the monocrotaline rat model of PAH.

With this study, benzbromarone becomes the latest addition to a long list of existing drugs, including dichloroacetate [16], tacrolimus [17], hydroxychloroquine [18] and others, which are being considered for repurposing as a PAH therapy. As reviewed recently by PRINS et al. [19], drug repurposing offers a great advantage for rare and deadly diseases like PAH by allowing for the fast-tracking of regulatory approval, while minimising the costs associated with new drug development. It is noteworthy that benzbromarone is the third gout treatment that has been considered for repurposing in $\mathrm{PAH}$, along with colchicine and 
allopurinol. Importantly, both allopurinol and benzbromarone are inhibitors of xanthine oxidase, raising the possibility that at least a portion of benzbromarone's therapeutic benefit, beyond any actions on TMEM16A, may be attributable to its impact on the generation of reactive oxygen species (ROS) [20].

When considering the potential repurposing of any drug for use in $\mathrm{PAH}$, it is essential that the candidate demonstrate both safety and efficacy in the human patient population. Regarding the former, benzbromarone may be at a disadvantage relative to other repurposing candidates. Although benzbromarone is still available as a generic drug in several countries, concerns over hepatotoxicity caused Sinofi-Synthélabo to withdraw the drug from the market in 2003. A subsequent risk-benefit analysis suggested that these safety concerns may have been overblown [21]. However, in order to be clinically viable, benzbromarone must still demonstrate sufficient efficacy to outweigh any potential risks to patient safety. In this regard, it is important to acknowledge that benzbromarone was efficacious in preventing the onset of disease in multiple rodent models, but has not yet been shown to reverse established disease in these models, or in the sugen-hypoxia rat model of $\mathrm{PAH}$, which has now become the gold standard pre-clinical model for the translation of potential PAH therapies [17, 22].

Despite the absence of these data, PAPP et al. [13] did examine the capacity of benzbromarone to induce an acute vasodilatory response in a small group of $10 \mathrm{PAH}$ patients. In these subjects, the administration of $200 \mathrm{mg}$ benzbromarone, twice the standard daily dose for the treatment of gout, failed to reduce mean pulmonary arterial pressure or pulmonary vascular resistance and actually caused a small but significant increase in both values. Perhaps this result is not surprising when considering previous clinical experiences with L-type, voltage-dependent calcium channel blockers (CCBs). CCBs, such as amlodipine, nifedipine or diltiazem, only induce an acute haemodynamic response in a subset of IPAH patients [23] and are effective for chronic administration in just a small fraction of these individuals, representing the 5-10\% of $\mathrm{PAH}$ patients for whom excessive and chronic vasoconstriction is a dominant feature of their disease [24]. Although chronic administration of benzbromarone may be required to achieve a beneficial effect in the broader PAH population, it is also possible that targeting altered anion handling may only be a viable therapeutic strategy for a small percentage of $\mathrm{PAH}$ patients. If this latter situation is the case, one must also consider whether this subset would represent the same group of individuals that is already clinically well served by CCBs, or if targeting altered anion flux will open up new therapeutic options for a currently undefined set of patients.

Independent of the ultimate clinical value of benzbromarone as a therapeutic strategy, it is worthwhile noting that, with the adoption of CCBs and sildenafil as approved therapies, $\mathrm{PAH}$ is already a drug repurposing success story. Moreover, all other approved treatments for PAH originated as designated orphan drugs, dating back to the first use of epoprostenol in 1984 [25]. This history exemplifies the advantages of drug repurposing and expedited drug designations as mechanisms to provide therapeutic options for a relatively small patient group in desperate need of treatment. However, this track record is also a reminder that the field has yet to develop an approved therapy that was custom designed exclusively for $\mathrm{PAH}$, based on the unique molecular pathobiology driving disease progression.

With the rise of high-throughput DNA sequencing technology, a number of new PAH-associated genes have recently been identified to accompany the initial identification of mutations in BMPR2, the gene encoding the bone morphogenetic protein (BMP) type II receptor (BMPR-II), nearly 20 years ago [26-30]. These genes have served to identify novel dysregulated signalling pathways in disease and shed light on the cellular processes that govern the initiation and progression of PAH. More importantly, these new targets also offer the promise of a next generation of tailor-made molecular therapies that are grounded not just in animal models or the cellular phenotype of patients with established PAH, but in the genetics underlying the human condition. Interestingly, a number of these newly identified genes include components of the BMPR-II signalling pathway, such as the BMPR-II ligands BMP9 and BMP10 [28-30], reinforcing the importance of this receptor to disease and supporting the pursuit of therapeutic approaches that aim to restore dysfunctional BMP signalling in PAH. As this work towards the next generation of $\mathrm{PAH}$ therapies proceeds, the question remains whether such custom therapeutics are required to truly change the clinical landscape or whether the recycling of drugs that were developed to treat other, unrelated pathologies represents the preferred path forward.

Conflict of interest: None declared.

Support statement: This work was supported by the Canadian Institutes of Health Research (grant PJT-152916). Funding information for this article has been deposited with the Crossref Funder Registry.

\section{References}

1 Hong Z, Chen KH, DasGupta A, et al. MicroRNA-138 and microRNA-25 down-regulate mitochondrial calcium uniporter, causing the pulmonary arterial hypertension cancer phenotype. Am J Respir Crit Care Med 2017; 195: 515-529. 
2 Bonnet S, Rochefort G, Sutendra G, et al. The nuclear factor of activated $\mathrm{T}$ cells in pulmonary arterial hypertension can be therapeutically targeted. Proc Natl Acad Sci USA 2007; 104: 11418-11423.

3 Yuan J, Aldinger A, Juhaszova $\mathrm{M}$, et al. Dysfunctional voltage-gated $\mathrm{K}^{+}$channels in pulmonary artery smooth muscle cells of patients with primary pulmonary hypertension. Circulation 1998; 98: 1400-1406.

4 Yu Y, Keller SH, Remillard CV, et al. A functional single-nucleotide polymorphism in the TRPC6 gene promoter associated with idiopathic pulmonary arterial hypertension. Circulation 2009; 119: 2313-2322.

5 Yuan XJ, Wang J, Juhaszova $\mathrm{M}$, et al. Attenuated $\mathrm{K}^{+}$channel gene transcription in primary pulmonary hypertension. Lancet 1998; 351: 726-727.

6 Ma L, Roman-Campos D, Austin ED, et al. A novel channelopathy in pulmonary arterial hypertension. $N$ Engl J Med 2013; 369: 351-361.

7 Higasa K, Ogawa A, Terao C, et al. A burden of rare variants in BMPR2 and KCNK3 contributes to a risk of familial pulmonary arterial hypertension. BMC Pulm Med 2017; 17: 57.

8 Antigny F, Hautefort A, Meloche J, et al. Potassium channel subfamily K member 3 (KCNK3) contributes to the development of pulmonary arterial hypertension. Circulation 2016; 133: 1371-1385.

9 Olschewski A, Li Y, Tang B, et al. Impact of TASK-1 in human pulmonary artery smooth muscle cells. Circ Res 2006; 98: 1072-1080.

10 Forrest AS, Joyce TC, Huebner ML, et al. Increased TMEM16A-encoded calcium-activated chloride channel activity is associated with pulmonary hypertension. Am J Physiol Cell Physiol 2012; 303: C1229-C1243.

11 Sun H, Xia Y, Paudel O, et al. Chronic hypoxia-induced upregulation of Ca2+-activated Cl- channel in pulmonary arterial myocytes: a mechanism contributing to enhanced vasoreactivity. J Physiol (Lond) 2012; 590: 3507-3521.

12 Bertrand J, Dannhoffer L, Antigny F, et al. A functional tandem between transient receptor potential canonical channels 6 and calcium-dependent chloride channels in human epithelial cells. Eur J Pharmacol 2015; 765: 337-345.

13 Papp R, Nagaraj C, Zabini D, et al. Targeting TMEM16A to reverse vasoconstriction and remodelling in idiopathic pulmonary arterial hypertension. Eur Respir J 2019; 53: 1800965.

14 Sinclair DS, Fox IH. The pharmacology of hypouricemic effect of benzbromarone. J Rheumatol 1975; 2: 437-445

15 Huang F, Zhang $\mathrm{H}$, Wu M, et al. Calcium-activated chloride channel TMEM16A modulates mucin secretion and airway smooth muscle contraction. Proc Natl Acad Sci USA 2012; 109: 16354-16359.

16 Michelakis ED, Gurtu V, Webster L, et al. Inhibition of pyruvate dehydrogenase kinase improves pulmonary arterial hypertension in genetically susceptible patients. Sci Transl Med 2017; 9: eaao4583.

17 Spiekerkoetter E, Tian X, Cai J, et al. FK506 activates BMPR2, rescues endothelial dysfunction, and reverses pulmonary hypertension. J Clin Invest 2013; 123: 3600-3613.

18 Long L, Yang X, Southwood M, et al. Chloroquine prevents progression of experimental pulmonary hypertension via inhibition of autophagy and lysosomal bone morphogenetic protein type II receptor degradation. Circ Res 2013; 112: 1159-1170.

19 Prins KW, Thenappan T, Weir EK, et al. Repurposing medications for treatment of pulmonary arterial hypertension: what's old is new again. J Am Heart Assoc 2019; 8: e011343.

20 Hoshikawa Y, Ono S, Suzuki S, et al. Generation of oxidative stress contributes to the development of pulmonary hypertension induced by hypoxia. J Appl Physiol 2001; 90: 1299-1306.

21 Lee MH, Graham GG, Williams KM, et al. A benefit-risk assessment of benzbromarone in the treatment of gout. Was its withdrawal from the market in the best interest of patients? Drug Saf 2008; 31: 643-665.

22 Long L, Ormiston ML, Yang X, et al. Selective enhancement of endothelial BMPR-II with BMP9 reverses pulmonary arterial hypertension. Nat Med 2015; 21: 777-785.

23 Rich S, Brundage BH. High-dose calcium channel-blocking therapy for primary pulmonary hypertension: evidence for long-term reduction in pulmonary arterial pressure and regression of right ventricular hypertrophy. Circulation 1987; 76: 135-141.

24 Sitbon $\mathrm{O}$, Humbert $\mathrm{M}$, Jais $\mathrm{X}$, et al. Long-term response to calcium channel blockers in idiopathic pulmonary arterial hypertension. Circulation 2005; 111: 3105-3111.

25 Daizadeh I. Pulmonary arterial hypertension: a case study in FDA expedited program designations. Ther Innov Regul Sci 2019; 53: 264-269.

26 Lane KB, Machado RD, Pauciulo MW, et al. Heterozygous germline mutations in BMPR2, encoding a TGF-beta receptor, cause familial primary pulmonary hypertension. The International PPH Consortium. Nat Genet 2000; 26: 81-84.

27 Deng Z, Morse JH, Slager SL, et al. Familial primary pulmonary hypertension (gene PPH1) is caused by mutations in the bone morphogenetic protein receptor-II gene. Am J Hum Genet 2000; 67: 737-744.

28 Eyries M, Montani D, Nadaud S, et al. Widening the landscape of heritable pulmonary hypertension mutations in paediatric and adult cases. Eur Respir J 2019; 53: 1801371.

29 Graf S, Haimel M, Bleda M, et al. Identification of rare sequence variation underlying heritable pulmonary arterial hypertension. Nat Commun 2018; 9: 1416.

30 Wang XJ, Lian TY, Jiang X, et al. Germline BMP9 mutation causes idiopathic pulmonary arterial hypertension. Eur Respir J 2019; 53: 1801609. 